\title{
Effects of Deposition Conditions on Hydrous Ruthenium Oxides Coatings on Ti Substrate for Supercapacitors by Cathodic Deposition Method
}

\author{
Yuli Lin and H.-S Hwang \\ Department of Mechanical Engineering \\ Chung Hua University, Hsinchu, 300 Taiwan ROC
}

Supercapacitors have many advantages using in electrical devices for their larger capacitance - high power density and long cycle life [1]. In general, supercapacitors can be classified into two categories, namely, pseudo-capacitors and double-layer capacitors. The fomer stores electrical charges in electrode surface region by faradaic reaction. While, in the latter, electrical charges are stored ate the double-layer formed at electrode/electrolyte interface. Various methods have been utilized to manufacture electrode including cyclic voltammetric method sol-gel method 、 anodizing - cathodic deposition method and etc.[2].

In this study, hydrous ruthenium oxide was deposited on Ti substrate by cathodic deposition method. Ti substrate was first cleaned thoroughly by acetone and followed by chemical etching of 5\%HF for 5 minutes and $50 \% \mathrm{HCl}$ for 15 minutes. These processes will produce many intensive voids which increase the mechanical lacking of the coating layer. The specimen was then immersed into the coating bath. The electrical capacity characteristics of coating were examined by cyclic voltammetry. The effects of electric capacity upon changing the concentration of ethanol and interfacial activated agents were also investigated. The microstructure of hydrous ruthenium oxide coating was also observed by scanning electron microscopy.

Figure 1(a) and (b) illustrates the microstructure after chemical etching of $\mathrm{Ti}$ substrate. It demonstrates that microvoids were produced on the surface of Ti substrate. Figure 2 shows the surface structure of hydrous ruthenium oxide coating. The electrical capacitance of specimen without any additives was measured about $255 \mathrm{~F} / \mathrm{g}$. It was observed that the electrical capacitance of specimen with ethanol added in the deposition bath was measured about $428 \mathrm{~F} / \mathrm{g}$. Figure 3(a) reveals the difference of electrical capacity characteristics of specimens with or without ethanol added in deposition processes. Obviously, ethanol added in the deposition bath will increase tremendously on the electrical electrical capacity characteristics. The reason could be that ethanol increase the effective current during deposition which leads the increasing of the amount of hydrous ruthenium oxide onto the Ti substrate. However, it was investigated that he electrical capacitance of specimen with interfacial activated agent (Triton X-100) added in the deposition bath was measured about 343F/g. Figure 3(b) demonstrates the difference of electrical capacity characteristics of specimens with or without Triton X-100 added during deposition processes. Although added of interfacial activated agent can increase the amount of hydrous ruthenium oxide onto the Ti substrate, the electrical capacitance was found decreasing.

[1] H.-S Hwang and Yuli Lin, Chung Hua Journal of Science and Engineering, Vol. 2, No.3, p7986(2004).

[2] H.-S Hwang, Master Thesis, Chung-Hua University, 2004. 
[3] This research was supported by NSC93-2216-E-216-009 and CHU93-2216-E-216-009.
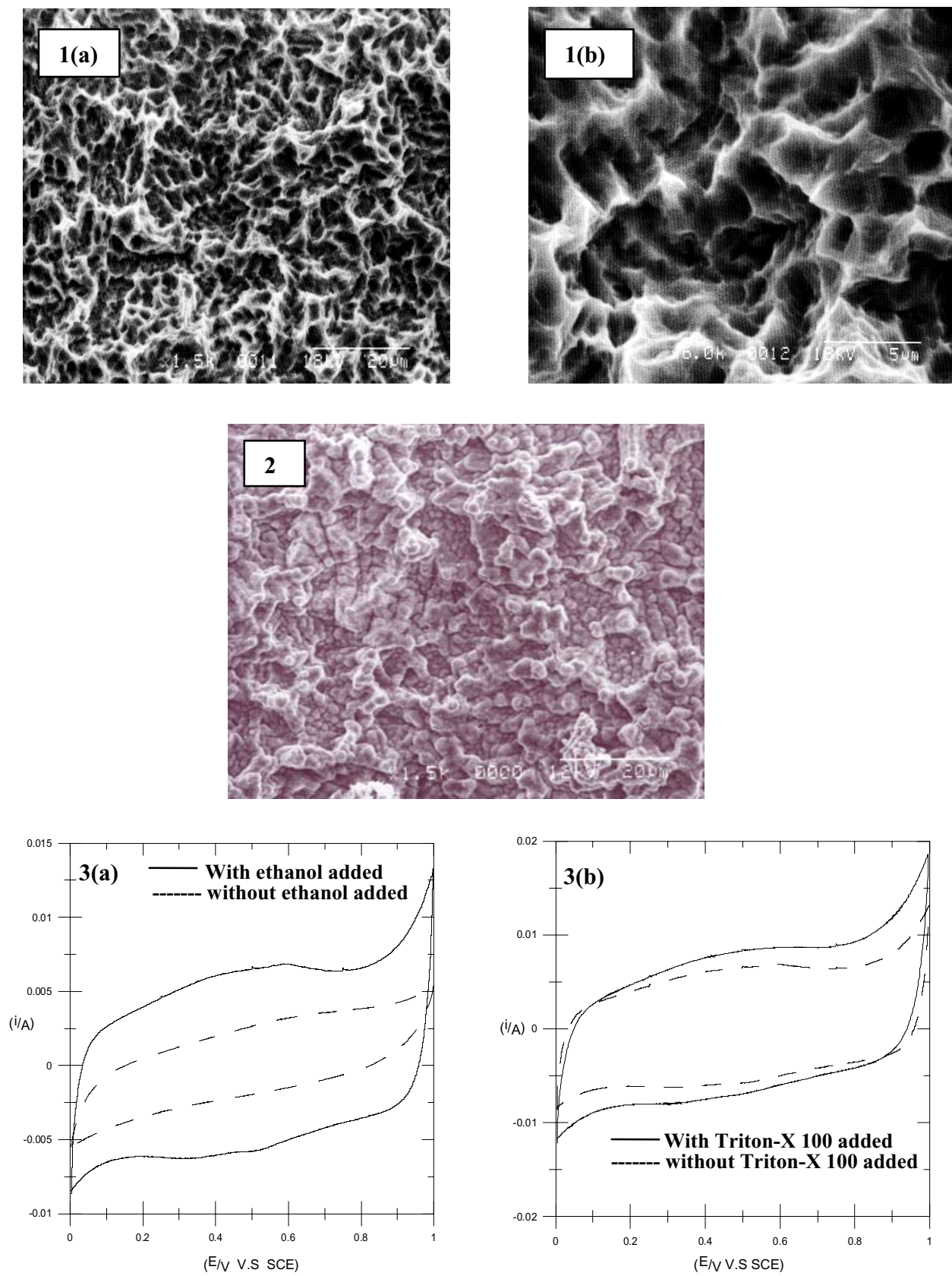

Fig.1 (a) The microstructure after chemical etching of Ti substrate demonstrating that microvoids were produced on the surface of Ti substrate.

(b) The microstructure after chemical etching of Ti substrate in higher magnification from (a).

Fig.2 Surface structure of hydrous ruthenium oxide coating without any additives.

Fig.3 (a) The difference of electrical capacity characteristics of specimens with or without ethanol added in deposition processes.

(b) The difference of electrical capacity characteristics of specimens with or without Triton X100 added during deposition processes. 